\title{
Efficient Uncertainty Quantification using Gradient-Enhanced Kriging
}

\author{
Richard P. Dwight*† and Zhong-Hua Han* \\ German Aerospace Center (DLR), D-38102 Braunschweig, Germany. \\ TU Delft, 2600GB Delft, The Netherlands.
}

\begin{abstract}
A flexible non-intrusive approach to parametric uncertainty quantification problems is developed, aimed at problems with many uncertain parameters, and for applications with a high cost of functional evaluations. It employs a Kriging response surface in the parameter space, augmented with gradients obtained from the adjoint of the deterministic equations. The Kriging correlation parameter optimization problem is solved using the Subplex algorithm, which is robust for noisy functionals, and whose effort typically increases only linearly with problem dimension. Integration over the resulting response surface to obtain statistical moments is performed using sparse grid techniques, which are designed to scale well with dimensionality. The efficiency and accuracy of the proposed method is compared with probabilistic collocation, direct application of sparse grid methods, and Monte-Carlo initially for model problems, and finally for a 2d compressible Navier-Stokes problem with a random geometry parameterized by 4 variables.
\end{abstract}

\section{Introduction}

Computational methods in science and engineering have become key technologies in a wide range of application areas. A notable example is Computational Fluid Dynamics (CFD) in transport and aviation, which is one of the most numerically and computationally demanding fields of modeling, but which nevertheless plays important roles at all stages of the modern aircraft design process and is widely accepted as an essential tool. However the application of uncertainty quantification in this field has been limited due to the high computational cost of performing deterministic calculations.

The problem we are concerned with here is to quantify parametric uncertainty, i.e. to determine the probability density function (PDF) (or statistical moments such as mean and variance), of some output quantity $J$ of a computational code, given a vector of stochastic input parameters $\alpha$ of size $M$, with known PDFs. So far, efforts in the literature have mainly concentrated on problems with only a few uncertain parameters. Due to the correspondence between the $M$ and the dimension of the spaces which arise, the problem becomes exponentially more demanding for increasing $M$ because of the curse of dimensionality i.e. the exponential growth of (hyper-)volume as a function of dimensionality. ${ }^{2}$

However complex multi-physics engineering problems routinely involve tens of physical and numerical parameters. Even for relatively simple geometries, taking into account shape variations for the purposes of shape optimization requires tens of parameters in $2 \mathrm{~d}$ and potentially hundreds is $3 \mathrm{~d} .{ }^{4}$ Furthermore the solution of the deterministic problem is often already an extremely computationally demanding task, and performing a large number of such solves is unrealistic. There is therefore a pressing need for efficient uncertainty quantification methods in very high dimensions.

Uncertainty quantification approaches may be divided into two broad classes: intrusive and non-intrusive, referring to whether the approach requires modification of the modeling code or not. The most widely-used intrusive technique, polynomial chaos, offers efficiency gains over the current leading non-intrusive methods, but implementation requires a very large code-specific and uncertain parameter-specific effort. Such an effort

*Institute of Aerodynamics and Flow Technology - Dept. Numerical Methods, Braunschweig.

${ }^{\dagger}$ Faculty of Aerospace - Aerodynamics Group, TU Delft. 